\title{
Contractile response to TRPA1 activation by allyl-isothiocyanate in isolated mouse distal colon: role of substance $P$ and CGRP released from TRPA1- expressing neurons
}

\author{
Kimihito Tashima, Yuki Goto, Yukiko Noma, Kazuki Hashimoto, Syunji Horie \\ Lab. of Pharmacology, Faculty of Pharmaceutical Sciences, Josai International Univ., Japan
}

BACKGROUND: TRPA1 channel, which was the first identified as a cold-sensitive cation channel in primary sensory neurons, responds to exogenous alimentary and environmental irritants so that TRPA1 is thought to be a chemosensor in gastrointestinal tract. Previously, we have shown that TRPA1 activation with allyl-isothiocyanate, a pungent ingredient of wasabi, induces contraction in isolated mouse distal colon, which is mediated by cholinergic neurons and tachykinincontaining nerve fibers pharmacologically (EJP 2007). In this study, we investigated (1) whether TRPA1 channels in the both neuronal and non-neuronal cells contribute to the contractile responses to allyl-isothiocyanate, and (2) if endogenous prostaglandins (PGs) produced by colonic epithelium are also involved in those contractions. METHODS: The distal colon was surgically isolated from male $\mathrm{ddY}$ mice. The longitudinal change in smooth muscle tension was isotonically measured using Magnus apparatus. In some experiments, preparations were pre-incubated for 5, 12, and 20 min with channel blockers, a calcitonin gene-related peptide (CGRP) receptor blocker BIBN 4096, and indomethacin before applied allyl-isothiocyanate. TRPA1 expression in the mouse distal colon was detected by immunohistochemical analysis. RESULTS: In the distal colon, the TRPA1 activator allyl-isothiocyanate induced transient contractions. The contractile response to allyl-isothiocyanate was markedly inhibited by a TRPA1 channel blocker A-967079, but not a TRPV1 channel blocker BCTC. The allyl-isothiocyanate-induced contraction in the distal colon was obviously inhibited by a sodium channel blocker tetrodotoxin. Those contractions induced by allyl-isothiocyanate were inhibited by BIBN 4096 and indomethacin. In the immunohistochemical study, TRPA1 immunoreactivity was found in the submucosal and muscle layers, and myenteric plexus in the transverse section of mouse distal colon. Several TRPA1 nerve fibers were observed to contain CGRP and substance P (SP). CONCLSION: These results suggest that contractile response to TRPA1 activation with allyl-isothiocyanate were mediated by CGRP and SP released from TRPA1-expressing neurons, which are localized in the mouse distal colon. In addition, it is suggested that the endogenous PGs play a role in the contraction in response to allyl-isothiocyanate by interacting with TRPA1-expressing neurons. TRPA1 channels may be one of important chemosensors that sense luminal environment, and then its activity is implicated in colonic motility. 